\title{
Dietary fatty acid intake, its food sources and determinants in European adolescents: the HELENA (Healthy Lifestyle in Europe by Nutrition in Adolescence) Study
}

Krishna E. Vyncke ${ }^{1,2 *}$, Lars Libuda ${ }^{3}$, Tineke De Vriendt ${ }^{1,2}$, Luis A. Moreno ${ }^{4}$, Myriam Van Winckel $^{5}$, Yannis Manios ${ }^{6}$, Frederic Gottrand ${ }^{7}$, Denes Molnar ${ }^{8}$, Barbara Vanaelst ${ }^{1,2}$, Michael Sjöström ${ }^{9}$, Marcela González-Gross ${ }^{10,11}$, Laura Censi ${ }^{12}$, Kurt Widhalm ${ }^{13}$, Nathalie Michels ${ }^{1}$, Chantal C. Gilbert ${ }^{14}$, Christos Xatzis $^{15}$, Magdalena Cuenca García ${ }^{16}$, Fátima Pérez de Heredia ${ }^{17}$, Stefaan De Henauw ${ }^{1}$ and Inge Huybrechts ${ }^{1}$ on behalf of the HELENA consortium $\dagger$

${ }^{1}$ Department of Public Health, Faculty of Medicine and Health Sciences, Ghent University, De Pintelaan $185-2$ Blok A, B-9000 Ghent, Belgium

${ }^{2}$ Research Foundation-Flanders, Egmontstraat 5, B-1000 Brussels, Belgium

${ }^{3}$ Research Institute of Child Nutrition, Dortmund, Germany

${ }^{4}$ GENUD (Growth, Exercise, Nutrition and Development) Research Group, Escuela Universitaria de Ciencias de la Salud,

University of Zaragoza, Zaragoza, Spain

${ }^{5}$ Department of Pediatrics and Medical Genetics, Ghent University Hospital, Ghent, Belgium

${ }^{6}$ Department of Nutrition and Dietetics, Harokopio University, Athens, Greece

${ }^{7}$ Inserm U995, IFR114, Faculté de Médecine, Université de Lille 2, Lille, France

${ }^{8}$ Department of Pediatrics, University of Pécs, Pécs, Hungary

${ }^{9}$ Department of Biosciences, Unit for Preventive Nutrition, Karolinska Institutet, Huddinge, Sweden

${ }^{10}$ Faculty of Physical Activity and Sport Sciences, University Polytechnic of Madrid, Madrid, Spain

${ }^{11}$ Department of Nutrition and Food Science, University of Bonn, Bonn, Germany

${ }^{12}$ INRAN (National Research Institute on Food and Nutrition), Rome, Italy

${ }^{13}$ Department of Pediatrics, Division of Clinical Nutrition and Prevention, Medical University of Vienna, Vienna, Austria

${ }^{14}$ Department of Consumer and Sensory Sciences, Campden BRI, Chipping Campden, Gloucestershire, UK

${ }^{15}$ Preventive Medicine and Nutrition Unit, University of Crete School of Medicine, Heraklion, Crete, Greece

${ }^{16}$ Department of Medical Physiology, Faculty of Medicine, Granada, Spain

${ }^{17}$ Immunonutrition Research Group, Department of Metabolism and Nutrition, Institute of Food Science and Technology and Nutrition (ICTAN), Spanish National Research Council (CSIC), Madrid, Spain

(Submitted 8 November 2011 - Final revision received 6 January 2012 - Accepted 16 January 2012 - First published online 28 February 2012)

\section{Abstract}

Dietary fatty acids (FA) play a role in several (patho)physiological processes at any age, and different FA have different effects on lipid status and health outcome. The present study aims to describe the FA intake and its main food sources in a population of healthy European adolescents and to assess the variation in intake as a function of non-dietary factors. FA intake was assessed with $24 \mathrm{~h}$ recall interviews in 1804 adolescents aged 12.5-17.5 years. Usual intakes were calculated using the multiple source method. Multilevel analyses, adjusting for study centre, were used to investigate the influence of non-dietary factors. The mean total fat intake was 33.3 (SD 1.2 ) \% of total energy intake $(\% \mathrm{E})$. The mean SFA intake was 13.8 ( $\mathrm{SD} 1.2$ ) \% , with $99.8 \%$ of the population exceeding the recommendations. SFA was mainly delivered by meat and cake, pies and biscuits. In most adolescents, the PUFA intake was too low, and $35.5 \%$ of the population did not achieve the minimum recommended intake for $\alpha$-linolenic acid (ALA). The main determinants of FA intake in the present study population were age and sex, as well as physical activity in the male subgroup. No contributions of body composition, socio-economic

Abbreviations: ALA, $\alpha$-linolenic acid; BLS, Bundeslebensmittelschlüssel (German Food Code and Nutrient Data Base); DPA, docosapentaenoic acid; $\% \mathrm{E}$, percentage of energy; FA, fatty acid; HELENA, Healthy Lifestyle in Europe by Nutrition in Adolescence; LA, linoleic acid; MSM, multiple source method; $\mathrm{P}_{5}$, 5th percentile; $\mathrm{P}_{95}$, 95th percentile.

*Corresponding author: Krishna Vyncke, fax +32933249 94, email Krishna.Vyncke@UGent.be

† See the Appendix for a full list of the HELENA study group members. 
status or sexual maturation to the variance in FA intake were observed. In conclusion, the most important public health concerns regarding FA intake in this adolescent population were the low intake of ALA and the high intake of SFA, mainly seen in the younger-aged boys. In this group the major contributor to SFA was meat.

\section{Key words: Fatty acids: Dietary intake: Food sources: Adolescents}

Diet and more specifically intake of fats and fatty acids (FA) have been extensively correlated with CVD risk ${ }^{(1)}$. Although CVD usually manifests in adulthood, cardiometabolic risk factors (such as hypertension, hyperlipidaemia, insulin resistance, obesity, etc.) may appear as early as childhood and adolescence $^{(2)}$. These risk factors may track into adulthood ${ }^{(3)}$, demonstrating the importance to direct research and intervention for CVD towards younger age groups such as adolescents. Apart from this well-documented association with CVD, dietary fats also play a role in the modulation of immune functions and inflammatory processes ${ }^{(4,5)}$, growth and development ${ }^{(6)}$, mental and neurodegenerative diseases and possibly in certain forms of cancer ${ }^{(4)}$

Studies assessing the intake of fats generally show that too high amounts of SFA and too low amounts of PUFA are consumed compared with current dietary guidelines ${ }^{(7-10)}$. Although blood lipids and FA are not only determined by their individual intake, most studies only assess the intake of total fat, SFA, PUFA, MUFA and cholesterol. However, it has been shown that different FA may have different effects on lipid status and health outcome. Whilst SFA with twelve to sixteen carbon atoms tend to increase plasma total and LDLcholesterol levels, stearic acid (18:0) does not have a cholesterol-raising effect ${ }^{(11)}$. Replacement of SFA in the diet by both $n-6$ or $n$-3 PUFA reduces the risk of $\mathrm{CVD}^{(12)}$ but the biological pathways behind these effects differ ${ }^{(13)}$. Regarding their influence on lipid status, $n-6$ FA have an LDL-cholesterol-lowering effect ${ }^{(14)}$ whilst $n-3$ FA reduce TAG levels ${ }^{(15)}$. In contrast, replacement of SFA by carbohydrates can even increase the risk of $\mathrm{CVD}^{(16)}$. Also sex differences have been reported regarding effects of dietary fat on CVD by lowering the HDL-cholesterol and increasing the levels of TAG ${ }^{(17)}$. As such, it is accepted that the food sources and type of fat consumed is most probably of greater importance than the total amount of fat, although the optimal intake of different FA remains unsettled ${ }^{(16,18)}$

Numerous dietary surveys have been conducted across Europe at national and local level; however, only a little information exists on the usual dietary intake of healthy adolescents. Furthermore, comparison between these studies is often difficult because of differences in dietary intake assessment methods ${ }^{(10)}$, failure to exclude under-reporters, lack of anthropometric data and varying age categories ${ }^{(19)}$. Besides, in different countries, various food composition tables are used for the conversion of food intake data into the estimated nutrient intakes, making comparisons difficult and inaccurate ${ }^{(10,19)}$.

To our knowledge, few studies have examined the influence of certain determinants on the intake of fat and individual FA. The objective of the present study was to describe the FA intake and its main food sources in a population of healthy
European adolescents and to study the variation in intake as a function of non-dietary factors such as sex, age, body composition, sexual maturation, socio-economic status and physical activity.

\section{Subjects and methods}

\section{Study design}

The 'Healthy Lifestyle in Europe by Nutrition in Adolescence (HELENA) Cross-Sectional Study' was set up to get an insight into the nutritional status and lifestyle habits of adolescents in order to permit further investigation of the associations between diet and health. The study aimed to obtain standardised, reliable and comparable data from a sample of European adolescents on a broad battery of relevant nutrition and health-related parameters $^{(20,21)}$. Data collection took place from November 2006 until December 2007, in ten conveniently selected European cities (Vienna, Ghent, Lille, Dortmund, Athens, Heraklion, Pécs, Rome, Zaragoza and Stockholm). A detailed description of the HELENA Cross-Sectional Study sampling and recruitment procedures, standardisation and harmonisation processes, data collection, analysis strategies and quality-control activities has been published elsewhere ${ }^{(22)}$.

\section{Subjects}

The adolescents, aged 12.5-17.5 years, were recruited from randomly selected schools in ten European cities. After receiving complete information about the aims and methods of the study, all adolescents and their parents or guardians signed, fully informed, a written consent. Participants were excluded a posteriori from the database if they met one of the exclusion criteria, namely age $<12.5$ or $\geq 17.5$ years, absence of data on weight and/or height, completion of $<75 \%$ of the tests, participating simultaneously in another clinical trial or having an acute infection lasting less than 1 week before the inclusion $^{(22)}$. The total eligible HELENA Cross-Sectional Study population consisted of 3528 adolescents (response rate $61.3 \%$ ). For the purpose of the present study, only adolescents with two $24 \mathrm{~h}$ dietary recall assessments were included in the analyses, resulting in 2330 cases. As such, all participants from Heraklion (Greece) and Pécs (Hungary) ( $n$ 678) were excluded from these analyses as no nutrient intake information could be calculated for these two cities. Therefore, only eight HELENA centres were included in the present study. Furthermore, exclusion of under-reporters resulted in a valid sample of 1804 adolescents (53\% females). Underreporting was considered when the individual ratio of energy intake:estimated BMR was lower than 0.96, as proposed by $\mathrm{Black}^{(23)}$. In this sample, information on physical 
activity was only available in 800 boys and 893 girls, resulting in a total, final study population of 1693 adolescents.

The study was performed following the ethical guidelines of the Declaration of Helsinki, the Good Clinical Practice rules, and the legislation about clinical research in human subjects in each of the participating countries. The protocol was approved by the Human Research Review Committees of the centres involved ${ }^{(24)}$

\section{Dietary intake assessment}

Following recommendations of the European Food Consumption Survey method (EFCOSUM) project, two non-consecutive $24 \mathrm{~h}$ recalls were completed by the adolescents ${ }^{(25)}$. The dietary intake assessment was performed by a computer-based tool for self-reported $24 \mathrm{~h}$ recalls, the HELENA Dietary Assessment Tool. This tool was based on a previous version developed for Flemish adolescents, called the Young Adolescents' Nutrition Assessment on Computer (YANA-C) ${ }^{(26)}$, which has been shown to provide a valid measurement of food consumption compared with an interview by a dietitian ${ }^{(27)}$. Food intake refers to the day before the $24 \mathrm{~h}$ recall assessment and is divided into six meal occasions. For each occasion the user is invited to select all the consumed food items and beverages from a standardised food list. Foods and beverages not included in the list can be added at all times. Information on quantities is gathered by use of household measurements or pictures of portion sizes. The self-administration took place in a computer classroom where the participants completed the program autonomously whilst field workers were present to give assistance if necessary ${ }^{(27)}$. The use of supplements was not evaluated as dietary intake.

Consumed foods were translated to nutrients by use of the German Food Code and Nutrient Data Base (Bundeslebensmittelschlüssel (BLS), version II.3.1) ${ }^{(28)}$. Although most of the recipes had been decomposed into ingredients, some complex foods that were available in the food composition table have been linked to the complex food directly (for example, coffee or tea with milk were linked to these complex foods directly, without disaggregation in coffee or tea and milk). The multiple source method (MSM), a statistical modelling technique, was used to estimate the usual dietary intake of nutrients and foods ${ }^{(29,30)}$. Comparison of the MSM to other conventional methods to estimate usual intake distributions (for example, Iowa State University method, National Cancer Institute method, Statistical Program for Age-adjusted Dietary Assessment) showed no important differences ${ }^{(31)}$. For nutrients, all estimates of the percentiles and mean were within $5 \%$ of each other ${ }^{(31)}$. As such, the European MSM was chosen, developed in the frame of the European Food Consumption Validation (EFCOVAL) project. This method has the advantage that the software is freely available and that covariates can be included. The MSM calculates dietary intake for individuals first and then constructs the population distribution based on the individual data. The method can make use of supporting data on consumption frequency; however, as only nutrient intakes were evaluated, and given that the same nutrients derive from different food sources, all participants can be considered daily consumers. Study centre, age, sex and its interaction term were taken into account in the calculations.

\section{Physical examination}

The protocol used to collect anthropometric data has been previously described ${ }^{(32)}$. Participants were barefoot and in underwear. Weight was measured with an electronic scale (SECA 861) to the nearest $0 \cdot 1 \mathrm{~kg}$. Height was measured in the Frankfort plane with a telescopic stadiometer (SECA 225) to the nearest $0 \cdot 1 \mathrm{~cm}$. BMI was calculated as body weight $(\mathrm{kg})$ divided by the square of height $(\mathrm{m})$. In addition, BMI was adjusted for age and sex to give a BMI standard deviation score using British 1990 growth reference data from the Child Growth Foundation ${ }^{(33)}$. Body fat percentage was calculated from the triceps and subscapular skin folds using the Slaughter formulae ${ }^{(34)}$, which seem to be the most suitable to be used in adolescents $^{(35)}$.

Pubertal status (stages I-V) was assessed by a medical doctor according to Tanner \& Whitehouse ${ }^{(36)}$, based on breast development and pubic hair status in females and genital and pubic hair development in males.

\section{Socio-economic status and physical activity}

Self-reported questionnaires were used to collect data on socio-economic status and physical activity. Maternal education level (lower education, secondary education and higher or university education) was chosen as a measure of socio-economic status of the adolescents. A measure for physical activity was obtained by the International Physical Activity Questionnaire for Adolescents (IPAQ-A). The validity of this questionnaire has been published previously ${ }^{(37)}$. The IPAQ-A covers four domains of physical activity: school-related physical activity (including activity during physical education classes and breaks), transportation, housework and activities during leisure time. Activities were afterwards classified into low, moderate and vigorous activity according to the guidelines for data processing and analyses of the IPAQ ${ }^{(38)}$. Total time spent on moderate and vigorous activity was summed and truncated in order to avoid overestimations ${ }^{(39)}$.

\section{Statistical analyses}

PASW 18.0 for Windows (SPSS, Inc.) was used. Characteristics of the study sample are presented as mean (standard deviation), unless otherwise stated. Baseline characteristics between the sexes were compared using a Student's $t$ test or Mann-Whitney $U$ test for continuous variables and the Pearson $\chi^{2}$ test for categorical variables.

Associations between the usual FA intake and its determinants (sex, age, body composition, sexual maturation, maternal education and physical activity) were tested with multilevel analysis to correct for the study design (clustering of cases within cities). FA intake was expressed as percentage of energy $(\% \mathrm{E})$ to correct for the varying total energy intake between different age groups and sex. Tests for normality were performed using the Kolmogorov-Smirnov test. Variables 
on usual intake were logarithmically transformed and geometrical means and $95 \%$ CI were reported. Because of physiological differences occurring during puberty, all values were presented stratified by sex. Differences between sexes within the same age group were assessed with a multilevel analysis stratified for age group. To correct for multiple testing a Bonferroni correction was applied; as such, a $P$ value of $0.003(0.05 /$ $18=0.003)$ was used as the threshold of significance and $P$ values between 0.003 and 0.01 were denoted as borderline significant. Two-sided significance levels were quoted.

The usual daily intakes of total fat, SFA and PUFA were compared with recommended population goals in adolescents. According to the recommendation of the $\mathrm{FAO}^{(4)}$, the selected goals were as follows: total fat, $25-35 \%$ of total energy intake (\%E); SFA, <8\%E; PUFA, 6-11\%E; linoleic acid (LA), $>2.5 \% \mathrm{E} ; \alpha$-linolenic acid (ALA), >0.5\%E. Differences between groups were assessed with a Pearson $\chi^{2}$ test.

The population proportion formula was used to determine the percentage contribution of food groups to the intake of fats and FA. This was done by summing the amount of the component provided by the food for all individuals divided by the total intake of that component from all foods for the entire study population ${ }^{(8,40,41)}$.

\section{Results}

The mean age was 14.7 (SD 1.2) years, with a median BMI of $20 \mathrm{~kg} / \mathrm{m}^{2}$; most participants (92\%) were categorised in Tanner stage 3 or higher. Significant sex differences were found for Tanner stage, with more males having a prepubertal Tanner stage. Characteristics of the study population for both sexes separately can be found in Table 1 .

The usual daily total fat intake ranged from $17 \cdot 8$ to $58 \cdot 9 \% \mathrm{E}$ (5th percentile $\left(\mathrm{P}_{5}\right) 25.7 \% \mathrm{E}$, 95th percentile $\left(\mathrm{P}_{95}\right) 41.6 \% \mathrm{E}$ ), with the lowest intake observed in the male study population and the highest intake in the female study population. The mean total fat intake was 33.3 (SD 1.2) \% E, with a mean SFA intake of $13 \cdot 8 \% \mathrm{E}\left(\mathrm{SD} 1 \cdot 2 ; \quad \mathrm{P}_{5} \quad 10 \cdot 1 \% \mathrm{E}, \mathrm{P}_{95} \quad 18 \cdot 2 \% \mathrm{E}\right)$, MUFA intake of $12 \cdot 2 \% \mathrm{E}$ (SD $1 \cdot 2 ; \mathrm{P}_{5} 9 \cdot 2 \% \mathrm{E}, \mathrm{P}_{95} 16 \cdot 3 \% \mathrm{E}$ ), and PUFA intake of $4.6 \% \mathrm{E}\left(\mathrm{SD} 1.3 ; \mathrm{P}_{5} 3.2 \% \mathrm{E}, \mathrm{P}_{95} 7 \cdot 0 \% \mathrm{E}\right.$ ). MUFA intake consisted mainly of oleic acid (mean $10 \cdot 7 \% \mathrm{E}$; SD $1 \cdot 2$; $\mathrm{P}_{5} 8.0 \% \mathrm{E}, \mathrm{P}_{95} 14.4 \% \mathrm{E}$ ). The geometric mean intake of lipids and FA in the male and female study population can be found in Table 2. In absolute amounts, the daily intake of fat and FA was, in general, significantly higher in the boys compared with the girls, except for EPA (boys $=56 \mathrm{mg} / \mathrm{d}$ and girls $=51 \mathrm{mg} / \mathrm{d} ; P=0 \cdot 020$ ) and DHA (boys $=133 \mathrm{mg} / \mathrm{d}$ and girls $=129 \mathrm{mg} / \mathrm{d} ; P=0 \cdot 383)$. However, when considering the $\% \mathrm{E}$ intake from fats and FA, the girls had significantly higher $\% \mathrm{E}$ intakes for all PUFA (boys $=4.4 \% \mathrm{E}$ and girls $=4.8 \% \mathrm{E} ; P<0.001)$ and had higher total fat intake of borderline significance (boys $=33.1 \% \mathrm{E}$ and girls $=33.7 \% \mathrm{E}$; $P=0 \cdot 009)$. No sex differences in MUFA and SFA intake were found, except for a borderline higher intake of lauric acid (12:0) (boys $=0.7 \% \mathrm{E}$ and girls $=0.7 \% \mathrm{E} ; P=0.005)$ in the female study sample. For the PUFA a higher intake of $n-6$ FA as well as $n$ - 3 FA was observed in the female study sample. The usual daily PUFA:SFA ratio was 0.33 in boys
Table 1. Basic characteristics of the study population

(Number of subjects and percentage; mean values and standard deviations; medians and ranges)

\begin{tabular}{|c|c|c|c|c|c|}
\hline & \multicolumn{2}{|c|}{ Boys } & \multicolumn{2}{|c|}{ Girls } & \multirow[b]{2}{*}{$P$} \\
\hline & $n$ & $\%$ & $n$ & $\%$ & \\
\hline Subjects & 855 & $47 \cdot 4$ & 949 & $52 \cdot 6$ & \\
\hline \multicolumn{6}{|l|}{ Age (years) ${ }^{*}$} \\
\hline Mean & \multicolumn{2}{|c|}{$14 \cdot 8$} & \multicolumn{2}{|c|}{$14 \cdot 7$} & 0.312 \\
\hline SD & \multirow{2}{*}{\multicolumn{2}{|c|}{$1 \cdot 3$}} & \multicolumn{2}{|c|}{$1 \cdot 2$} & \\
\hline \multicolumn{4}{|l|}{ BMI $\left(\mathrm{kg} / \mathrm{m}^{2}\right) \dagger$} & & \\
\hline Median & \multirow{2}{*}{\multicolumn{2}{|c|}{$\begin{array}{c}19 \cdot 9 \\
14 \cdot 2-35 \cdot 2\end{array}$}} & \multirow{2}{*}{\multicolumn{2}{|c|}{$\begin{array}{c}20 \cdot 2 \\
14 \cdot 1-40.8\end{array}$}} & 0.084 \\
\hline Range & & & & & \\
\hline \multicolumn{6}{|l|}{ SDS BMI* } \\
\hline Mean & \multicolumn{2}{|c|}{0.4} & \multicolumn{2}{|c|}{0.2} & 0.001 \\
\hline SD & \multicolumn{2}{|c|}{$1 \cdot 1$} & \multicolumn{2}{|c|}{1.0} & \\
\hline \multicolumn{6}{|c|}{ Body fat percentage†‡ } \\
\hline Median & \multirow{2}{*}{\multicolumn{2}{|c|}{$\begin{array}{c}16 \cdot 0 \\
6 \cdot 3-64 \cdot 6\end{array}$}} & \multirow{2}{*}{\multicolumn{2}{|c|}{$\begin{array}{c}24 \cdot 2 \\
10.7-54.8\end{array}$}} & $<0.001$ \\
\hline Range & & & & & \\
\hline \multicolumn{6}{|c|}{ Physical activity (min/week)†§ } \\
\hline Median & \multirow{2}{*}{\multicolumn{2}{|c|}{$\begin{array}{c}680 \cdot 0 \\
0.0-2520 \cdot 0\end{array}$}} & \multirow{2}{*}{\multicolumn{2}{|c|}{$\begin{array}{c}455.0 \\
0.0-2520.0\end{array}$}} & $<0.001$ \\
\hline Range & & & & & \\
\hline \multicolumn{6}{|l|}{ Tanner\|ף } \\
\hline Stage 1 and 2 & 94 & 11.4 & 45 & 4.9 & $<0.001$ \\
\hline Stage 3 & 200 & $24 \cdot 2$ & 232 & $25 \cdot 1$ & \\
\hline Stage 4 & 329 & $39 \cdot 8$ & 403 & $43 \cdot 6$ & \\
\hline Stage 5 & 204 & $24 \cdot 7$ & 245 & $26 \cdot 5$ & \\
\hline \multicolumn{6}{|c|}{ Education of mother $\|^{* *}$} \\
\hline Lower & 268 & $32 \cdot 7$ & 283 & $31 \cdot 3$ & 0.139 \\
\hline Secondary & 238 & 29 & 302 & 33.4 & \\
\hline Higher & 314 & $38 \cdot 3$ & 319 & $35 \cdot 3$ & \\
\hline
\end{tabular}

SDS, standard deviation score.

* Differences between the sexes were assessed with a Student's $t$ test.

$\dagger$ Differences between the sexes were assessed with a Mann-Whitney $U$ test.

‡Boys, $n$ 822; girls, $n 942$.

$\S$ Boys, $n$ 800; girls, $n 893$.

|| Differences between the sexes were assessed with a $\chi^{2}$ test.

१ Boys, $n$ 827; girls, $n 925$.

${ }^{\star \star}$ Boys, $n$ 820; girls, $n 904$.

and 0.36 in girls $(P<0.001)$. The ratio of $n-6: n-3$ FA intake was 5.95 in the total study sample and did not differ significantly between boys and girls; the intake of LA compared with ALA was 6.9:1.

Up to $57 \cdot 4 \%$ of the study population ( $58.4 \%$ of the boys; $56.5 \%$ of the girls; $P=0.420$ ) met the recommendations of the FAO for total fat intake. In $39 \%$ of the adolescents, total fat intake was higher than $35 \% \mathrm{E}$, and $3.6 \%$ had a usual intake lower than $25 \% \mathrm{E}$. SFA intake generally exceeded the dietary guidelines of $<8 \% \mathrm{E}$ in almost all adolescents (99.8\%) whilst PUFA intake was mostly too low, with only $12.7 \%$ of the adolescents adhering to the recommendations (6-11\%E). More girls than boys were in compliance with the PUFA guideline $(10.3 \%$ of the boys; $15 \%$ of the girls; $P=0.003)$. Whilst most adolescents (95.7\%) had an adequate intake of $>2.5 \% \mathrm{E}$ of LA to prevent deficiency symptoms, only $64.5 \%$ of the adolescents $58.5 \%$ of the boys; $70 \%$ of the girls; $P<0.001)$ achieved the minimum intake values for ALA $(>0.5 \% \mathrm{E})$, with the lowest intake in the study population being $0 \cdot 24 \% \mathrm{E}$.

Table 3 presents the percentage contributions of the major food groups to the usual lipid and FA intake in the total study population. The major source of total fat was meat ( $23.7 \%)$, followed by cakes, pies and biscuits (12.8\%), and cheese (7.4\%). 
Table 2. Usual fatty acid intake of European adolescents

(Geometric means and $95 \%$ confidence intervals)

\begin{tabular}{|c|c|c|c|c|c|}
\hline & \multicolumn{2}{|c|}{ Boys ( $n 800)$} & \multicolumn{2}{|c|}{ Girls ( $n$ 893) } & \multirow[b]{2}{*}{$P^{*}$} \\
\hline & Mean & $95 \% \mathrm{Cl}$ & Mean & $95 \% \mathrm{Cl}$ & \\
\hline Total fat $(\mathrm{g} / \mathrm{d})$ & 98.73 & $93.75,103.96$ & 78.59 & $74 \cdot 65,82 \cdot 74$ & $<0.001$ \\
\hline $\operatorname{SFA}(\mathrm{g} / \mathrm{d})$ & 41.05 & $38.91,43.32$ & 32.27 & $30.59,34.05$ & $<0.001$ \\
\hline Lauric acid (12:0) (g/d) & 2.05 & $1 \cdot 88,2 \cdot 25$ & 1.67 & $1.52,1.82$ & $<0.001$ \\
\hline Myristic acid $(14: 0)(\mathrm{g} / \mathrm{d})$ & 4.41 & $4 \cdot 16,4 \cdot 67$ & 3.52 & $3 \cdot 32,3 \cdot 74$ & $<0.001$ \\
\hline Palmitic acid $(16: 0)(\mathrm{g} / \mathrm{d})$ & $20 \cdot 37$ & $19 \cdot 35,21 \cdot 45$ & $16 \cdot 02$ & $15 \cdot 22,16 \cdot 86$ & $<0.001$ \\
\hline Stearic acid $(18: 0)(\mathrm{g} / \mathrm{d})$ & $9 \cdot 12$ & $8 \cdot 48,9.81$ & 7.07 & $6.58,7.60$ & $<0.001$ \\
\hline MUFA $(g / d)$ & $36 \cdot 26$ & $33.83,38.86$ & 28.87 & $26.94,30.93$ & $<0.001$ \\
\hline OA $(18: 1 n-9)(\mathrm{g} / \mathrm{d})$ & $31 \cdot 61$ & $29.40,33.99$ & $25 \cdot 20$ & $23.44,27.09$ & $<0.001$ \\
\hline PUFA (g/d) & 13.09 & $12 \cdot 18,14 \cdot 06$ & 11.09 & $10.32,11.91$ & $<0.001$ \\
\hline Total $n-6$ PUFA $(\mathrm{g} / \mathrm{d})$ & 11.06 & $10 \cdot 29,11.88$ & $9 \cdot 39$ & $8.74,10.09$ & $<0.001$ \\
\hline LA $(18: 2 n-6)(g / d)$ & $10 \cdot 65$ & $9 \cdot 88,11 \cdot 48$ & 9.08 & $8.43,9.79$ & $<0.001$ \\
\hline AA $(20: 4 n-6)(\mathrm{g} / \mathrm{d})$ & 0.32 & $0.27,0.37$ & 0.24 & $0.21,0.28$ & $<0.001$ \\
\hline Total n-3 PUFA (g/d) & 1.89 & $1.72,2.07$ & 1.60 & $1.46,1.75$ & $<0.001$ \\
\hline $\operatorname{ALA}(18: 3 n-3)(\mathrm{g} / \mathrm{d})$ & 1.60 & $1.44,1.79$ & 1.33 & $1.19,1.49$ & $<0.001$ \\
\hline EPA $(20: 5 n-3)(\mathrm{g} / \mathrm{d})$ & 0.06 & $0.05,0.07$ & 0.05 & $0.04,0.06$ & NS \\
\hline DPA $(22: 5 n-3)(\mathrm{g} / \mathrm{d})$ & 0.02 & $0.01,0.02$ & 0.01 & $0.01,0.01$ & $<0.001$ \\
\hline DHA $(22: 6 n-3)(\mathrm{g} / \mathrm{d})$ & 0.13 & $0.11,0.17$ & 0.13 & $0.10,0.16$ & NS \\
\hline Cholesterol $(\mathrm{mg} / \mathrm{d})$ & $402 \cdot 10$ & $368.72,438.51$ & $317 \cdot 23$ & $290.92,345.91$ & $<0.001$ \\
\hline Total fat (\%E) & $33 \cdot 12$ & $31.91,34.37$ & 33.73 & $32 \cdot 51,35 \cdot 01$ & NS \\
\hline SFA (\%E) & $13 \cdot 77$ & $13.37,14.18$ & 13.85 & $13.45,14.26$ & NS \\
\hline Lauric acid (12:0) (\%E) & 0.69 & $0.64,0.74$ & 0.72 & $0.67,0.77$ & NS \\
\hline Myristic acid (14:0) (\%E) & 1.48 & $1.41,1.55$ & 1.51 & $1.45,1.58$ & NS \\
\hline Palmitic acid (16:0) (\%E) & $6 \cdot 83$ & $6.61,7.07$ & $6 \cdot 87$ & $6 \cdot 65,7 \cdot 11$ & NS \\
\hline Stearic acid (18:0) (\%E) & 3.06 & $2 \cdot 92,3 \cdot 20$ & 3.04 & $2.90,3.18$ & NS \\
\hline MUFA (\%E) & $12 \cdot 17$ & $11.37,13.02$ & $12 \cdot 39$ & $11 \cdot 59,13 \cdot 26$ & NS \\
\hline $\mathrm{OA}(18: 1 n-9)(\% \mathrm{E})$ & $10 \cdot 61$ & $9.88,11.39$ & $10 \cdot 82$ & $10 \cdot 08,11.61$ & NS \\
\hline PUFA (\%E) & 4.39 & $4 \cdot 16,4 \cdot 64$ & 4.76 & $4.51,5.03$ & $<0.001$ \\
\hline Total $n-6$ PUFA (\%E) & $3 \cdot 71$ & $3.52,3.91$ & 4.03 & $3.82,4.25$ & $<0.001$ \\
\hline LA $(18: 2 n-6)(\% \mathrm{E})$ & 3.57 & $3.38,3.77$ & 3.90 & $3.69,4.12$ & $<0.001$ \\
\hline AA $(20: 4 n-6)(\% \mathrm{E})$ & $0 \cdot 11$ & $0.09,0.13$ & $0 \cdot 11$ & $0.09,0.12$ & NS \\
\hline Total $n$-3 PUFA (\%E) & 0.63 & $0.58,0.69$ & 0.69 & $0.63,0.74$ & $<0.001$ \\
\hline ALA $18: 3 n-3(\% \mathrm{E})$ & 0.54 & $0.49,0.59$ & 0.57 & $0.52,0.63$ & $<0.001$ \\
\hline EPA $(20: 5 n-3)(\% \mathrm{E})$ & 0.02 & $0.02,0.02$ & 0.02 & $0.02,0.03$ & $<0.001$ \\
\hline DPA (22:5n-3) (\%E) & 0.01 & $0.00,0.01$ & 0.00 & $0.00,0.01$ & $<0.001$ \\
\hline DHA $(22: 6 n-3)(\% \mathrm{E})$ & 0.05 & $0.04,0.06$ & 0.06 & $0.04,0.07$ & $<0.001$ \\
\hline Cholesterol $(\mathrm{mg} / 1000 \mathrm{kcal}) \dagger$ & 149.99 & $134.66,167.07$ & $151 \cdot 3$ & $135.87,168.55$ & NS \\
\hline
\end{tabular}

This same order applied for SFA and MUFA intake. Furthermore, sauces were an important contributor for lauric acid, milk for myristic acid, and chocolate for stearic acid. For all these FA the most important food sources were identical and of similar magnitude for boys and girls (Supplemental Tables 1 and 2; see supplementary material available online at http://www. journals.cambridge.org/bjn). Generally, meat was a slightly higher contributor to these FA in boys whilst cakes, pies and biscuits as well as nuts and seeds seemed to be of a higher importance in girls. PUFA were mostly delivered by the group of meat, fish, eggs and its substitutes as well as by low-nutrient, energydense foods (mainly cakes, pies and biscuits, savoury snacks and chocolate). A similar contribution pattern was seen for the essential FA, LA and ALA. However, where dairy products contributed only $5.7 \%$ to the LA intake, they supplied $13.4 \%$ of the ALA intake.

The intake of arachidonic acid was mainly provided by meat (46.2\%) followed by cakes, pies and biscuits. For EPA and DHA the major contributors were fish products followed by meat. In the female sample these foods contributed $70 \cdot 2$ and $22.9 \%$ of the EPA intake, respectively, whilst in the male population they contributed 56.7 and $33.6 \%$, respectively. In contrast, the major food source of docosapentaenoic acid (DPA), another long-chain $n-3$ PUFA, was meat $(65.6 \%$ in boys and $50.9 \%$ in girls) followed by fish products $(28.9 \%$ in boys and $43.6 \%$ in girls). The major contributors to cholesterol were meat, eggs, and cakes, pies and biscuits.

The results of the multilevel analyses, investigating the contribution of potential socio-demographic determinants to the variance in fat intake, are presented in Table 4 . In the male study sample, a significantly higher $\% \mathrm{E}$ intake from total fat and PUFA was seen in the older age group compared with the younger age group, leading to an equal intake of total fat in boys and girls between 15 and 17.5 years old (boys $=33.6 \% \mathrm{E}$ and girls $=34.0 \% \mathrm{E} ; P=0.997$ ). In the older boys higher $\% \mathrm{E}$ intakes of the essential FA (LA and ALA) and lower intakes of DPA intake were observed. In girls a significantly lower arachidonic acid and cholesterol intake was 
Table 3. Percentage contributions of food groups to the intake of fats and fatty acids in European adolescents

\begin{tabular}{|c|c|c|c|c|c|c|c|c|c|c|c|c|c|c|c|c|c|c|}
\hline \multirow[b]{2}{*}{ Food group } & \multicolumn{2}{|c|}{ Total fat } & \multicolumn{2}{|c|}{ SFA } & \multicolumn{2}{|c|}{ Lauric acid } & \multicolumn{2}{|c|}{ Myristic acid } & \multicolumn{2}{|c|}{ Palmitic acid } & \multicolumn{2}{|c|}{ Stearic acid } & \multicolumn{2}{|c|}{ MUFA } & \multicolumn{2}{|c|}{ Oleic acid } & \multicolumn{2}{|c|}{ Cholesterol } \\
\hline & $\%$ & $\mathrm{R}$ & $\%$ & $\mathrm{R}$ & $\%$ & $\mathrm{R}$ & $\%$ & $\mathrm{R}$ & $\%$ & $\mathrm{R}$ & $\%$ & $\mathrm{R}$ & $\%$ & $\mathrm{R}$ & $\%$ & $\mathrm{R}$ & $\%$ & $\mathrm{R}$ \\
\hline Beverages & 2.43 & & $2 \cdot 34$ & & $2 \cdot 29$ & & $2 \cdot 24$ & & 2.56 & & $2 \cdot 13$ & & $2 \cdot 20$ & & $2 \cdot 16$ & & $2 \cdot 14$ & \\
\hline Water & 0.00 & & 0.00 & & 0.00 & & 0.00 & & 0.00 & & 0.00 & & 0.00 & & 0.00 & & 0.00 & \\
\hline Coffee and tea & 0.25 & & 0.34 & & 0.32 & & 0.49 & & 0.31 & & 0.24 & & 0.21 & & 0.20 & & $0 \cdot 17$ & \\
\hline Fruit and vegetable juices & 0.40 & & 0.20 & & 0.09 & & 0.05 & & 0.34 & & 0.09 & & 0.18 & & 0.18 & & 0.00 & \\
\hline Carbonated, soft, isotonic drinks & 0.06 & & 0.03 & & 0.02 & & 0.01 & & 0.05 & & 0.02 & & 0.01 & & 0.01 & & 0.00 & \\
\hline Alcoholic beverages & 0.03 & & 0.04 & & 0.20 & & 0.06 & & 0.02 & & 0.02 & & 0.02 & & 0.02 & & 0.02 & \\
\hline Soups and bouillons & 1.69 & & 1.73 & & 1.66 & & 1.63 & & 1.84 & & 1.76 & & 1.78 & & 1.75 & & 1.95 & \\
\hline Bread and cereals & $6 \cdot 32$ & & $5 \cdot 25$ & & 6.49 & & $5 \cdot 23$ & & $5 \cdot 37$ & & 4.52 & & $6 \cdot 40$ & & $6 \cdot 39$ & & 2.93 & \\
\hline Bread and rolls & 4.94 & 7 & $4 \cdot 27$ & 8 & $5 \cdot 69$ & 7 & 4.59 & 7 & $4 \cdot 28$ & 8 & 3.38 & 7 & 4.69 & 7 & 4.49 & 7 & $2 \cdot 33$ & 10 \\
\hline Breakfast cereals & 0.66 & & 0.40 & & 0.05 & & 0.08 & & 0.47 & & 0.68 & & 0.77 & & 0.88 & & 0.01 & \\
\hline Flour & 0.72 & & 0.58 & & 0.75 & & 0.56 & & 0.62 & & 0.46 & & 0.94 & & 1.02 & & 0.59 & \\
\hline Potatoes and grains & $5 \cdot 37$ & & 3.91 & & $4 \cdot 88$ & & $3 \cdot 62$ & & $4 \cdot 33$ & & $3 \cdot 13$ & & $4 \cdot 65$ & & 4.69 & & 7.98 & \\
\hline Rice and other grains & 1.23 & & 0.90 & & 0.72 & & 0.82 & & 1.05 & & 0.74 & & 1.59 & & 1.72 & & 0.63 & \\
\hline Starchy roots, potatoes & $2 \cdot 25$ & & 1.63 & & $3 \cdot 14$ & 9 & 1.40 & & 1.70 & & 1.39 & & 1.65 & & 1.60 & & 0.58 & \\
\hline Pasta & 1.89 & & 1.38 & & 1.02 & & 1.40 & & 1.58 & & 1.00 & & 1.41 & & 1.37 & & $6 \cdot 77$ & 4 \\
\hline Vegetables and fruits & 3.37 & & 2.55 & & 2.53 & & $2 \cdot 24$ & & 2.94 & & 2.07 & & 3.58 & & 3.78 & & 1.54 & \\
\hline Vegetables & 2.58 & 9 & $2 \cdot 14$ & 10 & $2 \cdot 38$ & 10 & $2 \cdot 12$ & 10 & $2 \cdot 28$ & 9 & 1.87 & 10 & $2 \cdot 90$ & 9 & 3.04 & 9 & 1.51 & \\
\hline Fruits & 0.54 & & 0.30 & & 0.14 & & 0.09 & & 0.49 & & 0.14 & & 0.21 & & 0.22 & & 0.02 & \\
\hline Olives and avocados & 0.25 & & 0.11 & & 0.01 & & 0.03 & & 0.17 & & 0.06 & & 0.47 & & 0.52 & & 0.01 & \\
\hline Dairy and soya products & 16.93 & & 23.07 & & $22 \cdot 39$ & & $32 \cdot 86$ & & $20 \cdot 38$ & & $17 \cdot 70$ & & 14.64 & & $14 \cdot 15$ & & 13.59 & \\
\hline White milk and buttermilk & 4.89 & 8 & 6.86 & 6 & 7.05 & 5 & $10 \cdot 22$ & 3 & 6.00 & 6 & 4.83 & 6 & $4 \cdot 18$ & 8 & 3.95 & 8 & 4.57 & 6 \\
\hline Yogurt and fromage blanc & $1 \cdot 31$ & & 1.85 & & 1.79 & & $2 \cdot 76$ & 9 & 1.62 & & 1.30 & & $1 \cdot 11$ & & 1.04 & & 1.09 & \\
\hline Milk and yogurt beverages & 1.71 & & 2.41 & 9 & 1.82 & & $2 \cdot 81$ & 8 & $2 \cdot 11$ & 10 & $2 \cdot 67$ & 9 & 1.49 & & 1.48 & & 1.14 & \\
\hline Soya beverages & 0.33 & & 0.12 & & 0.02 & & 0.03 & & $0 \cdot 16$ & & $0 \cdot 12$ & & 0.21 & & 0.23 & & 0.01 & \\
\hline Cheese & 7.44 & 3 & $10 \cdot 21$ & 3 & $9 \cdot 85$ & 3 & 14.94 & 2 & 9.02 & 3 & 7.41 & 4 & 6.56 & 3 & $6 \cdot 38$ & 4 & 4.50 & 7 \\
\hline Desserts and puddings, milk based & $1 \cdot 21$ & & 1.57 & & 1.82 & & 2.03 & & 1.42 & & 1.33 & & 1.05 & & 1.03 & & $2 \cdot 22$ & \\
\hline Desserts and puddings, soya based & 0.01 & & 0.01 & & 0.00 & & 0.01 & & 0.01 & & 0.01 & & 0.01 & & 0.01 & & 0.00 & \\
\hline Other milk products & 0.03 & & 0.04 & & 0.04 & & 0.06 & & 0.04 & & 0.03 & & 0.03 & & 0.03 & & 0.06 & \\
\hline Fat and oil & $7 \cdot 73$ & & $8 \cdot 36$ & & 8.04 & & 10.99 & & 7.92 & & $6 \cdot 66$ & & 7.83 & & 7.03 & & 3.83 & \\
\hline Margarine and lipids of mixed origins & $2 \cdot 33$ & 10 & 1.49 & & 1.49 & & 1.34 & & 1.69 & & 1.52 & & $2 \cdot 78$ & 10 & 2.41 & 10 & 0.63 & \\
\hline Butter and animal fats & 5.40 & 5 & $6 \cdot 87$ & 5 & $6 \cdot 55$ & 6 & 9.65 & 5 & $6 \cdot 23$ & 5 & $5 \cdot 14$ & 5 & 5.05 & 6 & 4.62 & 6 & $3 \cdot 20$ & 9 \\
\hline Meat, fish, eggs, meat alternative & $28 \cdot 74$ & & $23 \cdot 22$ & & 12.93 & & $12 \cdot 78$ & & $28 \cdot 12$ & & $27 \cdot 72$ & & 33.43 & & 34.01 & & $48 \cdot 87$ & \\
\hline Meat & $23 \cdot 69$ & 1 & $19 \cdot 84$ & 1 & $7 \cdot 81$ & 4 & $10 \cdot 11$ & 4 & $24 \cdot 36$ & 1 & 24.91 & 1 & $28 \cdot 14$ & 1 & 28.56 & 1 & 31.98 & 1 \\
\hline Fish products & 1.49 & & $1 \cdot 19$ & & $4 \cdot 11$ & 8 & 1.54 & & 1.05 & & 0.69 & & $1 \cdot 14$ & & 0.95 & & 4.75 & 5 \\
\hline Eggs & 1.82 & & 1.54 & & 0.77 & & 0.91 & & 1.91 & & 1.51 & & 1.93 & & 2.01 & & $12 \cdot 00$ & 3 \\
\hline Meat substitutes and vegetarian products & $0 \cdot 17$ & & $0 \cdot 10$ & & 0.06 & & 0.07 & & $0 \cdot 13$ & & 0.08 & & $0 \cdot 13$ & & 0.14 & & 0.06 & \\
\hline Nuts and seeds & 1.43 & & 0.47 & & 0.14 & & 0.11 & & 0.56 & & 0.44 & & 1.97 & & $2 \cdot 22$ & & 0.00 & \\
\hline Pulses & 0.14 & & 0.08 & & 0.04 & & 0.04 & & 0.11 & & 0.09 & & 0.12 & & 0.13 & & 0.08 & \\
\hline Low-nutrient, energy-dense foods & $29 \cdot 11$ & & 31.33 & & $40 \cdot 46$ & & 30.05 & & 28.39 & & 36.06 & & $27 \cdot 30$ & & $27 \cdot 77$ & & $19 \cdot 11$ & \\
\hline Cakes, pies, biscuits & $12 \cdot 76$ & 2 & 14.47 & 2 & $20 \cdot 01$ & 1 & $18 \cdot 29$ & 1 & $12 \cdot 86$ & 2 & $12 \cdot 03$ & 3 & $12 \cdot 16$ & 2 & 11.28 & 2 & $12 \cdot 20$ & 2 \\
\hline Savoury snacks & 1.99 & & $1 \cdot 11$ & & $2 \cdot 25$ & & 0.41 & & 1.38 & & 0.89 & & 0.62 & & 0.63 & & 0.07 & \\
\hline Sugar, honey, jam and syrup & 0.22 & & 0.30 & & 0.29 & & 0.43 & & 0.27 & & 0.22 & & 0.18 & & 0.17 & & 0.13 & \\
\hline Confectionery, non-chocolate & 0.20 & & $0 \cdot 10$ & & 0.18 & & 0.07 & & 0.09 & & 0.11 & & 0.32 & & 0.36 & & 0.02 & \\
\hline Chocolate & $6 \cdot 34$ & 4 & $7 \cdot 74$ & 4 & 1.63 & & 1.86 & & $6 \cdot 81$ & 4 & $17 \cdot 31$ & 2 & $6 \cdot 34$ & 4 & $7 \cdot 19$ & 3 & 0.68 & \\
\hline Sauces & 5.06 & 6 & 5.05 & 7 & 13.39 & 2 & 6.02 & 6 & 4.47 & 7 & 3.32 & 8 & 5.08 & 5 & 5.48 & 5 & 3.35 & 8 \\
\hline Creams & 0.43 & & 0.59 & & 0.68 & & 0.87 & & 0.52 & & 0.42 & & 0.36 & & 0.34 & & 0.47 & \\
\hline Miscellaneous & $2 \cdot 11$ & & 1.97 & & 2.03 & & $2 \cdot 10$ & & 1.99 & & 1.76 & & $2 \cdot 24$ & & $2 \cdot 32$ & & $2 \cdot 19$ & \\
\hline
\end{tabular}


BS British Journal of Nutrition

Table 3. Continued

\begin{tabular}{|c|c|c|c|c|c|c|c|c|c|c|c|c|c|c|c|c|c|c|}
\hline \multirow[b]{2}{*}{ Food group } & \multicolumn{2}{|c|}{ PUFA } & \multicolumn{2}{|c|}{$\begin{array}{c}\text { Total } n-6 \\
\text { PUFA }\end{array}$} & \multicolumn{2}{|c|}{ LA } & \multicolumn{2}{|c|}{$\mathrm{AA}$} & \multicolumn{2}{|c|}{$\begin{array}{c}\text { Total } n-3 \\
\text { PUFA }\end{array}$} & \multicolumn{2}{|c|}{ ALA } & \multicolumn{2}{|c|}{ EPA } & \multicolumn{2}{|c|}{ DPA } & \multicolumn{2}{|c|}{ DHA } \\
\hline & $\%$ & $\mathrm{R}$ & $\%$ & $\mathrm{R}$ & $\%$ & $\mathrm{R}$ & $\%$ & $\mathrm{R}$ & $\%$ & $\mathrm{R}$ & $\%$ & $\mathrm{R}$ & $\%$ & $\mathrm{R}$ & $\%$ & $\mathrm{R}$ & $\%$ & $\mathrm{R}$ \\
\hline Beverages & $2 \cdot 81$ & & $2 \cdot 69$ & & $2 \cdot 69$ & & $2 \cdot 70$ & & 3.48 & & 3.97 & & 1.40 & & $1 \cdot 12$ & & 1.21 & \\
\hline Water & 0.00 & & 0.00 & & 0.00 & & 0.00 & & 0.00 & & 0.00 & & 0.00 & & 0.00 & & 0.00 & \\
\hline Coffee and tea & 0.14 & & 0.12 & & 0.12 & & 0.00 & & 0.24 & & 0.29 & & 0.00 & & 0.00 & & 0.00 & \\
\hline Fruit and vegetable juices & $1 \cdot 18$ & & 1.08 & & 1.12 & & 0.00 & & 1.74 & & $2 \cdot 12$ & & 0.00 & & 0.00 & & 0.00 & \\
\hline Carbonated, soft, isotonic drinks & 0.18 & & 0.17 & & 0.18 & & 0.00 & & 0.25 & & 0.30 & & 0.00 & & 0.00 & & 0.00 & \\
\hline Alcoholic beverages & 0.01 & & 0.01 & & 0.01 & & 0.01 & & 0.01 & & 0.02 & & 0.00 & & 0.00 & & 0.01 & \\
\hline Soups and bouillons & 1.30 & & 1.31 & & $1 \cdot 26$ & & $2 \cdot 69$ & 6 & 1.24 & & 1.24 & & 1.40 & 4 & $1 \cdot 12$ & 3 & 1.20 & 7 \\
\hline Bread and cereals & 7.54 & & 7.92 & & $7 \cdot 78$ & & $13 \cdot 00$ & & $5 \cdot 40$ & & $6 \cdot 24$ & & 1.38 & & 0.93 & & 1.84 & \\
\hline Bread and rolls & $5 \cdot 88$ & 5 & $6 \cdot 11$ & 5 & 5.91 & 5 & $12 \cdot 73$ & 3 & 4.60 & 7 & 5.33 & 6 & $1 \cdot 18$ & 6 & 0.85 & 6 & 1.49 & 6 \\
\hline Breakfast cereals & 1.09 & & 1.22 & & 1.27 & & 0.05 & & 0.34 & & 0.41 & & 0.00 & & 0.00 & & 0.00 & \\
\hline Flour & 0.57 & & 0.59 & & 0.60 & & 0.22 & & 0.46 & & 0.50 & & 0.20 & 9 & 0.08 & & 0.35 & \\
\hline Potatoes and grains & $10 \cdot 03$ & & $10 \cdot 42$ & & $10 \cdot 75$ & & 1.68 & & 7.87 & & $9 \cdot 34$ & & 0.80 & & 0.86 & & 1.49 & \\
\hline Rice and other grains & 1.29 & & 1.36 & & 1.39 & & 0.58 & & 0.90 & & 0.88 & & 0.69 & 8 & 0.86 & 5 & $1 \cdot 10$ & 9 \\
\hline Starchy roots, potatoes & $5 \cdot 32$ & 6 & $5 \cdot 38$ & 6 & 5.57 & 6 & 0.23 & & $5 \cdot 01$ & 5 & $6 \cdot 11$ & 4 & 0.08 & & 0.00 & & 0.08 & \\
\hline Pasta & 3.42 & 8 & 3.68 & 8 & 3.79 & 8 & 0.87 & 10 & 1.96 & & $2 \cdot 35$ & & 0.03 & & 0.00 & & 0.31 & \\
\hline Vegetables and fruits & 4.76 & & 4.45 & & 4.58 & & 1.46 & & 6.47 & & 7.50 & & 1.80 & & 0.97 & & 1.99 & \\
\hline Vegetables & 3.00 & & 2.84 & & 2.90 & & 1.39 & 8 & 3.89 & 10 & 4.39 & 9 & 1.66 & 3 & 0.88 & 4 & 1.80 & 5 \\
\hline Fruits & 1.57 & & 1.41 & & 1.47 & & 0.00 & & 2.46 & & 3.00 & & 0.00 & & 0.00 & & 0.01 & \\
\hline Olives and avocados & 0.19 & & 0.20 & & 0.21 & & 0.07 & & 0.12 & & 0.11 & & $0 \cdot 14$ & & 0.09 & & $0 \cdot 18$ & \\
\hline Dairy and soya products & 6.39 & & 5.54 & & 5.72 & & 0.97 & & $11 \cdot 16$ & & 13.44 & & 0.19 & & 0.23 & & 1.35 & \\
\hline White milk and buttermilk & 1.44 & & $1 \cdot 14$ & & $1 \cdot 18$ & & 0.15 & & 3.06 & & 3.70 & 10 & 0.01 & & 0.01 & & 0.26 & \\
\hline Yogurt and fromage blanc & 0.37 & & 0.29 & & 0.30 & & 0.00 & & 0.83 & & 1.01 & & 0.00 & & 0.00 & & 0.00 & \\
\hline Milk and yogurt beverages & 0.44 & & 0.38 & & 0.39 & & 0.01 & & 0.80 & & 0.97 & & 0.04 & & 0.01 & & 0.04 & \\
\hline Soya beverages & 1.31 & & 1.39 & & 1.44 & & 0.00 & & 0.89 & & 1.09 & & 0.00 & & 0.00 & & 0.00 & \\
\hline Cheese & $2 \cdot 28$ & & 1.85 & & 1.91 & & 0.38 & & 4.68 & 6 & 5.68 & 5 & 0.14 & & 0.21 & 9 & 0.23 & \\
\hline Desserts and puddings, milk based & 0.51 & & 0.45 & & 0.46 & & 0.42 & & 0.86 & & 0.94 & & 0.00 & & 0.00 & & 0.80 & 10 \\
\hline Desserts and puddings, soya based & 0.03 & & 0.03 & & 0.03 & & 0.00 & & 0.02 & & 0.02 & & 0.00 & & 0.00 & & 0.00 & \\
\hline Other milk products & 0.01 & & 0.01 & & 0.01 & & 0.01 & & 0.02 & & 0.03 & & 0.00 & & 0.00 & & 0.02 & \\
\hline Fat and oil & 7.41 & & 7.57 & & 7.85 & & $0 \cdot 16$ & & 6.57 & & 7.99 & & $0 \cdot 10$ & & 0.06 & & 0.21 & \\
\hline Margarine and lipids of mixed origins & 4.29 & 7 & 4.61 & 7 & 4.78 & 7 & 0.06 & & 2.54 & & 3.09 & & 0.01 & & 0.00 & & 0.07 & \\
\hline Butter and animal fats & $3 \cdot 12$ & 10 & 2.96 & 10 & 3.07 & 10 & 0.10 & & 4.03 & 8 & 4.90 & 7 & 0.09 & & 0.06 & & 0.14 & \\
\hline Meat, fish, eggs, meat alternatives & $32 \cdot 81$ & & $32 \cdot 63$ & & 31.68 & & $54 \cdot 16$ & & $33 \cdot 78$ & & 21.51 & & $92 \cdot 34$ & & 94.93 & & 85.82 & \\
\hline Meat & $24 \cdot 34$ & 1 & $25 \cdot 30$ & 1 & $24 \cdot 38$ & 1 & $46 \cdot 17$ & 1 & 19.02 & 1 & $16 \cdot 11$ & 1 & $27 \cdot 80$ & 2 & 58.42 & 1 & $30 \cdot 81$ & 2 \\
\hline Fish products & $2 \cdot 77$ & & 1.40 & & $1 \cdot 26$ & & 4.49 & 4 & $10 \cdot 31$ & 2 & 0.85 & & 64.33 & 1 & $36 \cdot 36$ & 2 & 48.58 & 1 \\
\hline Eggs & 1.65 & & 1.48 & & 1.43 & & 3.31 & 5 & $2 \cdot 61$ & & 2.32 & & 0.07 & & 0.13 & 10 & $6 \cdot 38$ & 3 \\
\hline Meat substitutes and vegetarian products & 0.52 & & 0.55 & & 0.57 & & 0.03 & & 0.35 & & 0.42 & & 0.01 & & 0.02 & & 0.03 & \\
\hline Nuts and seeds & 3.23 & 9 & 3.60 & 9 & 3.73 & 9 & 0.08 & & 1.17 & & 1.42 & & 0.08 & & 0.00 & & 0.00 & \\
\hline Pulses & 0.30 & & 0.30 & & 0.31 & & 0.08 & & 0.32 & & 0.39 & & 0.05 & & 0.00 & & 0.02 & \\
\hline Low-nutrient, energy-dense foods & $28 \cdot 22$ & & $28 \cdot 76$ & & 28.94 & & $25 \cdot 85$ & & $25 \cdot 30$ & & 29.98 & & 2.00 & & 0.90 & & 6.08 & \\
\hline Cakes, pies, biscuits & 9.91 & 2 & 9.93 & 2 & 9.56 & 2 & $22 \cdot 13$ & 2 & 9.82 & 3 & 11.47 & 2 & 0.03 & & 0.25 & 8 & 3.95 & 4 \\
\hline Savoury snacks & 6.75 & 3 & 6.43 & 4 & $6 \cdot 65$ & 4 & 0.51 & & 8.57 & 4 & $10 \cdot 48$ & 3 & 0.00 & & 0.00 & & 0.01 & \\
\hline Sugar, honey, jam and syrup & 0.12 & & 0.11 & & 0.11 & & 0.04 & & 0.19 & & 0.23 & & 0.00 & & 0.00 & & 0.00 & \\
\hline Confectionery, non-chocolate & 0.20 & & 0.22 & & 0.23 & & 0.00 & & 0.06 & & 0.08 & & 0.00 & & 0.00 & & 0.00 & \\
\hline Chocolate & 2.56 & & $2 \cdot 88$ & & 2.99 & & 0.07 & & 0.77 & & 0.93 & & 0.00 & & 0.00 & & $0 \cdot 13$ & \\
\hline Sauces & 6.45 & 4 & 6.89 & 3 & $7 \cdot 10$ & 3 & 1.20 & 9 & 4.02 & 9 & 4.81 & 8 & 0.71 & 7 & 0.08 & & 0.49 & \\
\hline Creams & 0.13 & & 0.10 & & 0.10 & & 0.06 & & 0.27 & & 0.32 & & 0.02 & & 0.02 & & $0 \cdot 10$ & \\
\hline Miscellaneous & $2 \cdot 10$ & & $2 \cdot 20$ & & $2 \cdot 20$ & & 1.84 & 7 & 1.60 & & 1.66 & & 1.24 & 5 & 0.55 & 7 & 1.40 & 8 \\
\hline
\end{tabular}


Table 4. Fatty acid intake of European adolescents (percentage of energy; \%E) according to age category and sex (Geometric means and $95 \%$ confidence intervals)

\begin{tabular}{|c|c|c|c|c|c|c|c|c|c|c|}
\hline \multirow{3}{*}{ Age category (years)... } & \multicolumn{4}{|c|}{ Boys } & \multirow[b]{3}{*}{$P^{*}$} & \multicolumn{4}{|c|}{ Girls } & \multirow[b]{3}{*}{$P^{*}$} \\
\hline & \multicolumn{2}{|c|}{$12.50-<15.00(n 455)$} & \multicolumn{2}{|c|}{$15 \cdot 00-17 \cdot 50$ (n 345) } & & \multicolumn{2}{|c|}{$12 \cdot 50-<15.00(n 518)$} & \multicolumn{2}{|c|}{$15 \cdot 00-17 \cdot 50$ (n 375) } & \\
\hline & Mean & $95 \% \mathrm{Cl}$ & Mean & $95 \% \mathrm{Cl}$ & & Mean & $95 \% \mathrm{Cl}$ & Mean & $95 \% \mathrm{Cl}$ & \\
\hline Total fat (\%E) & 32.53 & $31.33,33.78$ & $33 \cdot 61$ & $32 \cdot 35,34.92$ & 0.002 & 33.73 & $32.39,35.13$ & 33.99 & $32.63,35.42$ & NS \\
\hline SFA (\%E) & 13.64 & $13 \cdot 19,14 \cdot 11$ & $13 \cdot 86$ & $13 \cdot 39,14 \cdot 36$ & NS & 13.86 & $13 \cdot 47,14 \cdot 27$ & 13.91 & $13.50,14.34$ & NS \\
\hline Lauric acid (12:0) (\%E) & 0.66 & $0.61,0.71$ & 0.74 & $0.68,0.80$ & $<0.001$ & 0.69 & $0.64,0.73$ & 0.75 & $0.71,0.81$ & $<0.001$ \\
\hline Myristic acid (14:0) (\%E) & 1.47 & $1.39,1.54$ & 1.49 & $1.42,1.57$ & NS & 1.50 & $1.43,1.57$ & 1.54 & $1.47,1.61$ & NS \\
\hline Palmitic acid (16:0) (\%E) & $6 \cdot 76$ & $6.53,7.01$ & 6.87 & $6 \cdot 63,7 \cdot 12$ & NS & 6.91 & $6 \cdot 67,7 \cdot 17$ & 6.87 & $6 \cdot 62,7 \cdot 13$ & NS \\
\hline Stearic acid (18:0) (\%E) & 3.06 & $2 \cdot 91,3.22$ & 3.03 & $2 \cdot 87,3 \cdot 19$ & NS & 3.07 & $2.95,3.21$ & 3.01 & $2 \cdot 89,3.14$ & NS \\
\hline MUFA (\%E) & 11.97 & $11 \cdot 23,12 \cdot 76$ & $12 \cdot 27$ & $11.51,13.09$ & NS & $12 \cdot 41$ & $11.52,13.37$ & $12 \cdot 49$ & $11.60,13.46$ & NS \\
\hline $\mathrm{OA}(18: 1 n-9)(\% \mathrm{E})$ & $10 \cdot 42$ & $9 \cdot 76,11 \cdot 13$ & 10.72 & $10.04,11.45$ & NS & $10 \cdot 82$ & $10 \cdot 00,11.71$ & 10.93 & $10 \cdot 10,11.83$ & NS \\
\hline PUFA (\%E) & 4.23 & $3.98,4.50$ & 4.58 & $4.30,4.87$ & $<0.001$ & 4.73 & $4.49,4.99$ & 4.83 & $4.58,5 \cdot 10$ & NS \\
\hline Total $n-6$ PUFA (\%E) & 3.57 & $3.36,3.79$ & 3.87 & $3.65,4 \cdot 12$ & $<0.001$ & 4.01 & $3.81,4.23$ & 4.09 & $3.88,4.31$ & NS \\
\hline LA $(18: 2 n-6)(\% \mathrm{E})$ & 3.43 & $3.23,3.64$ & 3.74 & $3.51,3.98$ & $<0.001$ & 3.88 & $3.68,4.09$ & 3.96 & $3.76,4 \cdot 18$ & NS \\
\hline AA $(20: 4 n-6)(\% \mathrm{E})$ & 0.11 & $0.09,0.13$ & 0.10 & $0.09,0.12$ & NS & 0.11 & $0.09,0.13$ & 0.10 & $0.08,0.12$ & $<0.001$ \\
\hline Total $n$-3 PUFA (\%E) & 0.62 & $0.57,0.67$ & 0.65 & $0.60,0.71$ & 0.001 & 0.68 & $0.63,0.74$ & 0.70 & $0.64,0.76$ & NS \\
\hline ALA $(18: 3 n-3)(\% E)$ & 0.52 & $0.47,0.57$ & 0.57 & $0.52,0.62$ & $<0.001$ & 0.56 & $0.51,0.62$ & 0.58 & $0.53,0.64$ & NS \\
\hline EPA (20:5n-3) (\%E) & 0.02 & $0.02,0.02$ & 0.02 & $0.01,0.02$ & NS & 0.02 & $0.02,0.03$ & 0.02 & $0.02,0.03$ & NS \\
\hline DPA (22:5n-3) (\%E) & 0.01 & $0.01,0.01$ & 0.00 & $0.00,0.01$ & 0.001 & 0.00 & $0.00,0.01$ & 0.00 & $0.00,0.00$ & NS \\
\hline DHA $(22: 6 n-3)(\% \mathrm{E})$ & 0.05 & $0.04,0.06$ & 0.04 & $0.03,0.05$ & NS & 0.06 & $0.04,0.07$ & 0.05 & $0.04,0.07$ & NS \\
\hline Cholesterol $(\mathrm{mg} / 1000 \mathrm{kcal}) \dagger$ & $150 \cdot 86$ & $135.08,168.48$ & 147.51 & $132 \cdot 03,164 \cdot 82$ & NS & $155 \cdot 54$ & $138.86,174.22$ & $146 \cdot 33$ & $130.60,163.95$ & $<0.001$ \\
\hline
\end{tabular}

OA, oleic acid; LA, linoleic acid; AA, arachidonic acid; ALA, $\alpha$-linolenic acid; DPA, docosapentaenoic acid.

*Comparison of geometric means between age groups within sex, assessed with a multilevel analysis corrected for study centre, including physical activity as an independent variable (NS, $P>0.003$ ).

$\dagger 1000 \mathrm{kcal}=4184 \mathrm{~kJ}$. 
observed in the older age group. The $\% \mathrm{E}$ intake of lauric acid was significantly higher in the oldest age group of both boys and girls $(P<0 \cdot 001)$.

Besides the differences between age groups, a higher physical activity was associated with a significant lower intake of total fat $(\beta-0.000015 ; P<0.001)$, total SFA $(\beta-0.000016$; $P=0.001)$, total MUFA $(\beta-0.000018 ; P<0.001)$, oleic acid ( $\beta-0.000018 ; \quad P<0.001)$, palmitic acid $(\beta-0.000016$; $P<0.001)$ and stearic acid $(\beta-0.000017 ; P=0.002)$ in the male population, whilst in girls no significant contribution of physical activity to the variance in FA intake was observed (data not shown).

Furthermore, no significant associations were found between FA intake and the other determinants (BMI, body fat percentage, socio-economic status and sexual maturation) after correction for age, sex and physical activity and application of the Bonferroni correction (data not shown).

\section{Discussion}

In accordance with previous studies, high mean intakes of total fat and SFA and low mean intakes of MUFA and PUFA were observed in our European adolescent study population $^{(7-10,19,42-46)}$. Across Europe, mean total fat intakes of $25 \cdot 5-44 \cdot 2 \% \mathrm{E}$ have been recorded, with generally higher intakes in the Southern countries and lower intakes in the Northern countries ${ }^{(9,19)}$. In the present study, the usual daily PUFA:SFA ratio was 0.33 in boys and 0.36 in girls. Only few studies report this ratio, making comparison difficult. If ratios were calculated from the provided means of PUFA and SFA, often higher ratios have been observed in the female population $^{(7,8)}$ whilst Samuelson et al. reported higher ratios in the male population ${ }^{(42)}$. Much higher PUFA:SFA ratios (exceeding $0.5)$ have been recorded in the USA and Mexico ${ }^{(47,48)}$. The proportion of $n$ - 6 PUFA: $n$ - 3 PUFA intake in the present study population was about $6: 1$ and the proportion of LA:ALA was about $7: 1$. These ratios were much lower than generally reported in Western countries ${ }^{(47,49)}$ and in Mexico $^{(48)}$, but still much higher than the ratio of $n-6: n-3$ of 0.8 which would seem optimal from an evolutionary point of view ${ }^{(49)}$. However, due to unconvincing evidence and conceptual limitation, the FAO judged that there is no rationale for setting a recommendation for the $n-6: n-3$ or LA:ALA ratio ${ }^{(4)}$. Also the meaning and usefulness of ratios has been questioned previously because ratios can disguise extremely low or high intakes ${ }^{(50)}$. Instead it was recommended by the UK Food Standards Agency to focus on the effects of absolute amounts of individual $n-6$ and $n-3 \mathrm{FA}^{(51)}$.

Compared with the recently published recommendations of the FAO for fats and FA intake, the present results suggested that about half of the European adolescents met the recommended intake for total fat, but for about $39 \%$ of the adolescents total fat intake was too high. According to our data, $3.6 \%$ of the study population had a usual fat intake of less than $25 \% \mathrm{E}$, which could render this group prone to have an inadequate consumption of fat-soluble vitamins ${ }^{(4)}$. A slightly higher intake of total fat was observed in the female population due to a higher intake of MUFA and PUFA in comparison with boys. However, mean PUFA intake did not meet the recommendation for either sex. Differences in intake pattern between the sexes disappeared with older age except for PUFA intake, which continued to be higher in the female population. A high proportion $(35.5 \%)$ of the adolescents did not meet the minimum intake value for ALA $(>0.5 \% \mathrm{E})$. ALA is, however, indispensable since man cannot synthesise it and major deficiencies of this essential $n-3$ FA can result in skin changes, abnormal visual function and peripheral neuropathy ${ }^{(52)}$. This indicates the need to further stress the importance of a sufficient intake of this essential FA.

Similar to other studies ${ }^{(7,8,10)}$, almost all adolescents (99.8\%) exceeded the FAO recommendation of $8 \% \mathrm{E}$ for SFA intake. Even if the old recommendation of $\leq 10 \% \mathrm{E}$ is used, only $4.4 \%$ of the population was in compliance with the guideline. It is generally accepted that an excessive intake of SFA results in long-term health complications. However, as discussed in the 'Great Fat Debate', the proportion of energy from saturated fat has little effect on disease risk when the replacement nutrient is not specified ${ }^{(12,13,16,53,54)}$. Replacing SFA by PUFA has been shown to reduce the risk on CVD, but replacement by carbohydrates is unlikely to have any major effect on CVD risk, whilst replacement with highly refined carbohydrates may increase CVD risk ${ }^{(12)}$. Furthermore, evidence even suggests a potentially protective effect of saturated fat consumption for stroke risk ${ }^{(55)}$. Also, different SFA have different effects on plasma lipid levels and risk of $\mathrm{CVD}^{(18)}$. As such, myristic acid appears to raise total cholesterol in a more potent way than lauric acid and even more than palmitic acid, whilst stearic acid has no cholesterol-raising effect ${ }^{(11)}$ In the present study sample, as well as in others ${ }^{(42,47)}$, the intake of palmitic and stearic acids accounted for more than $70 \%$ of the total SFA intake, making it questionable whether these intakes might be tolerable. However, there are still insufficient data on the long-term health effects and usual intake of these individual FA to establish guidelines for these nutrients.

Comparison of the main contributors of food groups to nutrient intake is difficult because food groupings often differ. However, the top three contributors to total fat, SFA, MUFA and PUFA intake were meat, dairy products, and cakes, pies and biscuits, which is in agreement with other studies $^{(7,8,46)}$. In contrast to the present results, Joyce et al ${ }^{(7)}$ reported a higher contribution of whole milk than of cheese. The higher contribution of meat in boys compared with girls has also been reported previously ${ }^{(46)}$. As described by others $^{(8,41,47)}$, the main source of EPA and DHA came from fish intake, followed by meat. In contrast to previous assumptions $^{(41,56,57)}$, the present results showed that DPA intake was primarily provided by meat. This has also been reported by others ${ }^{(8,47)}$ and is most probably due to the high consumption rate of meat compared with fish.

Only a few studies have investigated the influence of nondietary factors on FA intake. The main determinants of FA intake in the present study population were age and sex. Chronological age was a stronger determinant of FA intake than sexual maturation. In the male population, fat and FA intake were also inversely related to their level of physical activity. Measures of body composition were not related to the usual fat and FA intake, and no differences in fat intake 
as a function of socio-economic status, assessed by the educational level of the mother, were observed. In contrast, Joyce $e t a l .{ }^{(7)}$ found that adolescents whose parents had secondary education had significantly higher \%E from MUFA than those whose parents had tertiary education. Furthermore, some studies showed an influence of a rural compared with an urban lifestyle ${ }^{(7,58)}$. This could not be evaluated in the present study as our total population was sampled from urban schools. In the present study, boys had a significantly lower intake of $\% \mathrm{E}$ from total PUFA than girls, which has also been described by Joyce et al. ${ }^{(7)}$ for Irish children and adolescents. In contrast to our findings, Samuelson et al. found a significantly higher \%E intake from PUFA and LA, and higher, although not significantly, intake of total fat, SFA and MUFA in 15-year-old boys compared with age-matched girls $^{(42)}$. These results were, however, based on a small sample size (forty-two and fifty-one, respectively). A significantly higher intake of LA was also found for boys in a group of 1117 American adolescents ${ }^{(43)}$. Furthermore, in the present study, girls had a significantly higher intake of the $n-3$ precursor ALA and of long-chain-PUFA (EPA and DHA), when expressed in $\% \mathrm{E}$; this difference has not been found by others ${ }^{(42,43)}$. In boys aged $15-17.5$ years compared with boys aged 12.5-14.9 years, a higher intake in \%E from total fat and PUFA was found. Other studies, on the contrary, have shown a tendency of decreasing intakes of these FA with increasing age in Irish and German adolescents ${ }^{(7,45)}$. In addition, older boys had a higher intake of the essential FA (LA and ALA) and lauric acid and a lower intake of the $n$ - 3 long-chain-PUFA. In summary, girls showed a more beneficial FA intake pattern; however, differences between boys and girls became less apparent or disappeared with increasing age.

\section{Strengths and limitations}

Being part of a multicentre European study, data were collected in a standardised way, following strict procedures. The analyses were performed on a group of adolescents of different ethnicities, with data available on anthropometrics, lifestyle factors and socio-economic status. The adolescents were randomly selected in ten European cities. Based on the HELENA key variables, no signs of selection bias were identified between participants and non-participants within these cities (L Béghin, I Huybrechts, G Vicente-Rodríguez, S De Henauw, F Gottrand, M González-Gross, J Dallongeville, M Sjöström, C Leclercq, S Dietrich, M Castillo, M Plada, D Molnar, M Kersting, CC Gilbert and LA Moreno, unpublished results); thus, the study sample seems to be representative for an urban European adolescent population. However, the selection of the cities was done in a convenient way, based on geographical distribution and the presence of an active research group. This selection was representative of the average level of demography, cultural, social and economic markers. The towns were equivalent and comparable between countries and their size was sufficiently large to ensure diversity. Still, as in all epidemiological studies, selection bias is difficult to identify and therefore cannot be ruled out.
A number of validation studies have shown that misreporting is a major problem in dietary surveys of adolescents. Following recommendations of the European Food Consumption Survey method (EFCOSUM), $24 \mathrm{~h}$ recalls were preferred because these are open-ended questionnaires in which detailed information can be obtained. A limitation of the method used is that only information of $2 \mathrm{~d}$ was obtained. As such, the dietary intake is prone to exceptional intakes. The $24 \mathrm{~h}$ dietary recall method does not allow quantifying proportions of non-consumers for particular food items, especially for infrequently consumed foods. To decrease this influence, days of assessment were randomly selected and nutrient intakes were corrected for within-individual variability by applying the MSM method. As only nutrient intakes were studied and not food intake, the influence of non-consumption days of certain food items (for example, fish) was attenuated. Still, as fish is a major contributor of EPA and DHA, an underestimation cannot be ruled out. However, the present study aimed to assess the mean FA intake in the total population and not the exact intake for each individual. Due to the high number of participants, variations by chance in individuals' long-chain-PUFA intake were attenuated. Furthermore, the obtained mean intake results are in line with the literature ${ }^{(10,41,44)}$.

Another limitation of the $24 \mathrm{~h}$ recalls is that data collection relies on the individual's ability to remember foods and beverages consumed in the past $24 \mathrm{~h}$; accuracy might therefore be biased towards under-reporting. In this respect, the $24 \mathrm{~h}$ dietary recalls were performed through computer-assisted HELENA Dietary Assessment Tool software to standardise the recall procedures as much as possible. Moreover, there is a potential loss of dietary information from mixed dishes, as food ingredients were sometimes counted from mixed dishes. Also, some information on food intake is less detailed, as adolescents are not acquainted with the applied food preparation methods and thereby used cooking fats. As such, average values and types of cooking fats used were estimated according to the countries' habitual preparation methods.

The same food composition table for conversion of food intake data to estimated nutrient intakes was used for all survey centres. In this way, differences in definitions, analytical methods, units and modes of expression were overcome. In this regard, the German food composition tables (BLS) were chosen. The BLS is based on German, American, English, Swedish, Danish and Dutch food composition tables, on analytical values of food-producing firms, publications and research results of the federal research centres and universities ${ }^{(59)}$. The BLS includes about 11000 raw and cooked foods and recipes and is widely used in epidemiological studies. Furthermore, the BLS is one of the tables giving the most complete coverage of the individual $\mathrm{FA}^{(60)}$. The FA composition of foods is based on analysis or estimation. Therefore, the intake of the different FA is only an approximation for a single individual. However, as stated above, in the present study, only the mean intake values of FA in the complete study sample were assessed. Therefore, the impact of potential errors is minimised due to the high number of study participants. 
It is noteworthy that the linking between the food consumption data and the BLS was done on the level of complex foods instead of their ingredients for some particular food items or drinks (for example, coffee or tea with milk). This should be considered when interpreting the contribution of food items and drinks to the different FA (for example, part of the contribution of coffee and tea to the FA intakes might come from the milk that was added to them).

\section{Conclusion}

In general, girls showed a more beneficial FA intake pattern than boys. However, differences between boys and girls became less apparent or disappeared with increasing age. Furthermore, in boys but not in girls, physical activity appeared to be a determinant of fat intake. The most important public health concerns regarding fat intake in this adolescent population were the low intake of ALA and the high intake of SFA, mainly palmitic and stearic acids. This was primarily seen in the younger-aged boys, where the major contributor to SFA was meat. Changing meat consumption to fish would substantially lower the SFA intake. However, at the same time, meat was also an important dietary source of DPA. As such, more information on the individual health effects of the different $\mathrm{FA}$ is needed to elucidate their importance and to set goals for these individual FA.

\section{Acknowledgements}

The present study was done as part of the HELENA study (www.helenastudy.com). We gratefully acknowledge the financial support of the European Community Sixth RTD Framework Programme (contract FOOD-CT-2005-007034). K. E. V., T. D. V. and B. V. are financially supported by the Research Foundation - Flanders. L. A. M., Y. M., F. G., D. M., M. S., M. G.-G., K. W., C. G. and S. D. H. designed the research. I. H. performed the data cleaning and K. E. V. did the statistical analysis. K. E. V., L. L., T. D. V., M. V. W., B. V., L. C., N. M., M. C. G. and F. P. H. wrote the paper and C. X. edited the manuscript. K. E. V. had primary responsibility for the final content. All authors read and approved the final manuscript. None of the authors had a personal or financial conflict of interest. The contents of this paper reflect only the authors' views and the rest of the HELENA study members are not responsible for it.

\section{APPENDIX: HELENA Study Group}

Co-ordinator: Luis A. Moreno.

Core Group members: Luis A. Moreno, Fréderic Gottrand, Stefaan De Henauw, Marcela González-Gross, Chantal Gilbert.

Steering Committee: Anthony Kafatos (President), Luis A. Moreno, Christian Libersa, Stefaan De Henauw, Sara Castelló, Fréderic Gottrand, Mathilde Kersting, Michael Sjöstrom, Dénes Molnár, Marcela González-Gross, Jean Dallongeville, Chantal Gilbert, Gunnar Hall, Lea Maes, Luca Scalfi.

Project Manager: Pilar Meléndez.
Universidad de Zaragoza (Spain): Luis A. Moreno, Jesús Fleta, José A. Casajús, Gerardo Rodríguez, Concepción Tomás, María I. Mesana, Germán Vicente-Rodríguez, Adoración Villarroya, Carlos M. Gil, Ignacio Ara, Juan Revenga, Carmen Lachen, Juan Fernández Alvira, Gloria Bueno, Aurora Lázaro, Olga Bueno, Juan F. León, Jesús Mำ Garagorri, Manuel Bueno, Juan Pablo Rey López, Iris Iglesia, Paula Velasco, Silvia Bel, Luis A. Gracia Marco, Theodora Mouratidou.

Consejo Superior de Investigaciones Científicas (Spain): Ascensión Marcos, Julia Wärnberg, Esther Nova, Sonia Gómez, Ligia Esperanza Díaz, Javier Romeo, Ana Veses, Belén Zapatera, Tamara Pozo, David Martínez.

Université de Lille 2 (France): Laurent Beghin, Christian Libersa, Frédéric Gottrand, Catalina Iliescu, Juliana Von Berlepsch.

Research Institute of Child Nutrition Dortmund, Rheinische Friedrich-Wilhelms-Universität Bonn (Germany): Mathilde Kersting, Wolfgang Sichert-Hellert, Ellen Koeppen.

Pécsi Tudományegyetem (University of Pécs) (Hungary): Dénes Molnar, Eva Erhardt, Katalin Csernus, Katalin Török, Szilvia Bokor, Enikö Nagy, Orsolya Kovács, Judit Répasi.

University of Crete School of Medicine (Greece): Anthony Kafatos, Caroline Codrington, María Plada, Angeliki Papadaki, Katerina Sarri, Anna Viskadourou, Christos Hatzis, Michael Kiriakakis, George Tsibinos, Constantine Vardavas, Manolis Sbokos, Eva Protoyeraki, Maria Fasoulaki.

Institut für Ernährungs- und Lebensmittelwissenschaften Ernährungphysiologie, Rheinische Friedrich-Wilhelms-Universität (Germany): Peter Stehle, Klaus Pietrzik, Marcela González-Gross, Christina Breidenassel, Andre Spinneker, Jasmin Al-Tahan, Miriam Segoviano, Anke Berchtold, Christine Bierschbach, Erika Blatzheim, Adelheid Schuch, Petra Pickert.

University of Granada (Spain): Manuel J. Castillo, Ángel Gutiérrez, Francisco B. Ortega, Jonatan R. Ruiz, Enrique G. Artero, Vanesa España, David Jiménez-Pavón, Palma Chillón, Cristóbal Sánchez-Muñoz, Magdalena Cuenca.

Istituto Nazionalen di Ricerca per gli Alimenti e la Nutrizione (Italy): Davide Arcella, Elena Azzini, Emma Barison, Noemi Bevilacqua, Pasquale Buonocore, Giovina Catasta, Laura Censi, Donatella Ciarapica, Paola D'Acapito, Marika Ferrari, Myriam Galfo, Cinzia Le Donne, Catherine Leclercq, Giuseppe Maiani, Beatrice Mauro, Lorenza Mistura, Antonella Pasquali, Raffaela Piccinelli, Angela Polito, Raffaella Spada, Stefania Sette, Maria Zaccaria, Romana Roccaldo.

University of Napoli 'Federico II' Department of Food Science (Italy): Luca Scalfi, Paola Vitaglione, Concetta Montagnese.

Ghent University (Belgium): Ilse De Bourdeaudhuij, Stefaan De Henauw, Tineke De Vriendt, Lea Maes, Christophe Matthys, Carine Vereecken, Mieke de Maeyer, Charlene Ottevaere, Inge Huybrechts.

Medical University of Vienna (Austria): Kurt Widhalm, Katharina Phillipp, Sabine Dietrich, Birgit Kubelka, Marion Boriss-Riedl.

Harokopio University (Greece): Yannis Manios, Eva Grammatikaki, Zoi Bouloubasi, Tina Louisa Cook, Sofia Eleutheriou, Orsalia Consta, George Moschonis, Ioanna Katsaroli, George Kraniou, Stalo Papoutsou, Despoina Keke, Ioanna Petraki, Elena Bellou, Sofia Tanagra, Kostalenia Kallianoti, 
Dionysia Argyropoulou, Katerina Kondaki, Stamatoula Tsikrika,

Christos Karaiskos.

Institut Pasteur de Lille (France): Jean Dallongeville, Aline Meirhaeghe.

Karolinska Institutet (Sweden): Michael Sjöstrom, Jonatan R. Ruiz, Francisco B. Ortega, María Hagströmer, Anita Hurtig Wennlöf, Lena Hallström, Emma Patterson, Lydia Kwak, Julia Wärnberg, Nico Rizzo.

Asociación de Investigación de la Industria Agroalimentaria (Spain): Jackie Sánchez-Molero, Sara Castelló, Elena Picó, Maite Navarro, Blanca Viadel, José Enrique Carreres, Gema Merino, Rosa Sanjuán, María Lorente, María José Sánchez.

Campden BRI (United Kingdom): Chantal Gilbert, Sarah

Thomas, Elaine Allchurch, Peter Burgess.

SIK - Institutet foer Livsmedel och Bioteknik (Sweden):

Gunnar Hall, Annika Astrom, Anna Sverkén, Agneta Broberg.

Meurice Recherche \& Development asbl (Belgium): Annick Masson, Claire Lehoux, Pascal Brabant, Philippe Pate, Laurence Fontaine.

Campden and Chorleywood Food Development Institute (Hungary): Andras Sebok, Tunde Kuti, Adrienn Hegyi.

Productos Aditivos SA (Spain): Cristina Maldonado, Ana Llorente.

Cárnicas Serrano SL (Spain): Emilio García.

Cederroth International AB (Sweden): Holger von Fircks, Marianne Lilja Hallberg, Maria Messerer.

Lantmännen Food RED (Sweden): Mats Larsson, Helena Fredriksson, Viola Adamsson, Ingmar Börjesson.

European Food Information Council (Belgium): Laura Fernández, Laura Smillie, Josephine Wills.

Universidad Politécnica de Madrid (Spain): Marcela González-Gross, Jara Valtueña, David Jiménez-Pavón, Ulrike Albers, Raquel Pedrero, Agustín Meléndez, Pedro J. Benito, Juan José Gómez Lorente, David Cañada, Alejandro Urzanqui, Rosa María Torres, Paloma Navarro.

\section{References}

1. Hu FB \& Willett WC (2002) Optimal diets for prevention of coronary heart disease. JAMA 288, 2569-2578.

2. Berenson GS, Srinivasan SR \& Nicklas TA (1998) Atherosclerosis: a nutritional disease of childhood. Am J Cardiol 82, 22T-29T.

3. Camhi SM \& Katzmarzyk PT (2010) Tracking of cardiometabolic risk factor clustering from childhood to adulthood. Int J Pediatr Obes 5, 122-129.

4. Food and Agriculture Organization (2010) FAO Report of an Expert Consultation on Fats and Fatty Acids in Human Nutrition. Rome: FAO.

5. Calder PC (2009) Fatty acids and immune function: relevance to inflammatory bowel diseases. Int Rev Immunol 28, 506-534.

6. Innis SM (2007) Dietary ( $n$-3) fatty acids and brain development. J Nutr 137, 855-859.

7. Joyce T, Wallace AJ, McCarthy SN, et al. (2009) Intakes of total fat, saturated, monounsaturated and polyunsaturated fatty acids in Irish children, teenagers and adults. Public Health Nutr 12, 156-165.
8. O'Sullivan TA, Ambrosini G, Beilin LJ, et al. (2011) Dietary intake and food sources of fatty acids in Australian adolescents. Nutrition 27, 153-159.

9. Elmadfa I, Meyer A, Nowak V, et al. (2009) European Nutrition and Health Report 2009. Ann Nutr Metab 55, Suppl. 2, $1-40$.

10. Harika RK, Cosgrove MC, Osendarp SJ, et al. (2011) Fatty acid intakes of children and adolescents are not in line with the dietary intake recommendations for future cardiovascular health: a systematic review of dietary intake data from thirty countries. Br J Nutr 106, 307-316.

11. Kris-Etherton PM \& Yu S (1997) Individual fatty acid effects on plasma lipids and lipoproteins: human studies. $A m J$ Clin Nutr 65, 1628S-1644S.

12. Mozaffarian D (2011) The great fat debate: taking the focus off of saturated fat. J Am Diet Assoc 111, 665-666.

13. Kuller LH (2011) The great fat debate: reducing cholesterol. J Am Diet Assoc 111, 663-664.

14. Rassias G, Kestin M \& Nestel PJ (1991) Linoleic acid lowers LDL cholesterol without a proportionate displacement of saturated fatty acid. Eur J Clin Nutr 45, 315-320.

15. Harris WS (1989) Fish oils and plasma lipid and lipoprotein metabolism in humans: a critical review. J Lipid Res 30, $785-807$.

16. Willett WC (2011) The great fat debate: total fat and health. $J$ Am Diet Assoc 111, 660-662.

17. Mozaffarian D, Rimm EB \& Herrington DM (2004) Dietary fats, carbohydrate, and progression of coronary atherosclerosis in postmenopausal women. Am J Clin Nutr 80, 1175-1184.

18. Hu FB, Manson JE \& Willett WC (2001) Types of dietary fat and risk of coronary heart disease: a critical review. $\mathrm{J} \mathrm{Am}$ Coll Nutr 20, 5-19.

19. Lambert J, Agostoni C, Elmadfa I, et al. (2004) Dietary intake and nutritional status of children and adolescents in Europe. Br J Nutr 92, Suppl. 2, S147-S211.

20. De Henauw S, Gottrand F, De Bourdeaudhuij I, et al. (2007) Nutritional status and lifestyles of adolescents from a public health perspective. The HELENA Project - Healthy Lifestyle in Europe by Nutrition in Adolescence. J Public Health 15 , 187-197.

21. Moreno LA, González-Gross M, Kersting M, et al. (2008) Assessing, understanding and modifying nutritional status, eating habits and physical activity in European adolescents: The HELENA (Healthy Lifestyle in Europe by Nutrition in Adolescence) Study. Public Health Nutr 11, 288-299.

22. Moreno LA, De Henauw S, González-Gross M, et al. (2008) Design and implementation of the Healthy Lifestyle in Europe by Nutrition in Adolescence Cross-Sectional Study. Int J Obes (Lond) 32, Suppl. 5, S4-S11.

23. Black AE (2000) Critical evaluation of energy intake using the Goldberg cut-off for energy intake:basal metabolic rate. A practical guide to its calculation, use and limitations. Int J Obes Relat Metab Disord 24, 1119-1130.

24. Beghin L, Castera M, Manios Y, et al. (2008) Quality assurance of ethical issues and regulatory aspects relating to good clinical practices in the HELENA Cross-Sectional Study. Int J Obes (Lond) 32, Suppl. 5, S12-S18.

25. Biro G, Hulshof KF, Ovesen L, et al. (2002) Selection of methodology to assess food intake. Eur J Clin Nutr $\mathbf{5 6}$, Suppl. 2, S25-S32.

26. Vereecken CA, Covents M, Matthys C, et al. (2005) Young Adolescents' Nutrition Assessment on Computer (YANA-C). Eur J Clin Nutr 59, 658-667.

27. Vereecken CA, Covents M, Sichert-Hellert W, et al. (2008) Development and evaluation of a self-administered compu- 
terized 24-h dietary recall method for adolescents in Europe. Int J Obes (Lond) 32, Suppl. 5, S26-S34.

28. Dehne LI, Klemm C, Henseler G, et al. (1999) The German Food Code and Nutrient Data Base (BLS II.2). Eur J Epidemiol 15, 355-359.

29. Haubrock J, Hartigg U, Souverein O, et al. (2010) An improved statistical tool for estimating usual intake distributions: the multiple source method (MSM). Arch Public Health 68, 14-15.

30. Department of Epidemiology of the German Institute of Human Nutrition Potsdam-Rehbrücke (DiFE) (2011) The Multiple Source Method (MSM). https://nugo.dife.de/msm

31. Souverein OW, Dekkers AL, Geelen A, et al. (2011) Comparing four methods to estimate usual intake distributions. EurJ Clin Nutr 65, Suppl. 1, S92-S101.

32. Nagy E, Vicente-Rodriguez G, Manios Y, et al. (2008) Harmonization process and reliability assessment of anthropometric measurements in a multicenter study in adolescents. Int $J$ Obes (Lond) 32, Suppl. 5, S58-S65.

33. Cole TJ, Freeman JV \& Preece MA (1995) Body mass index reference curves for the UK, 1990. Arch Dis Child 73, 25-29.

34. Slaughter M, Lohman T, Boileau R, et al. (1988) Skinfold equations for estimation of body fatness in children and youths. Hum Biol 60, 709-723.

35. Rodriguez G, Moreno LA, Blay MG, et al. (2005) Body fat measurement in adolescents: comparison of skinfold thickness equations with dual-energy X-ray absorptiometry. Eur J Clin Nutr 59, 1158-1166.

36. Tanner JM \& Whitehouse RH (1976) Clinical longitudinal standards for height, weight, height velocity, weight velocity, and stages of puberty. Arch Dis Child 51, 170-179.

37. Hagstromer M, Bergman P, De Bourdeaudhuij I, et al. (2008) Concurrent validity of a modified version of the International Physical Activity Questionnaire (IPAQ-A) in European adolescents: The HELENA Study. Int J Obes (Lond) 32, S42-S48.

38. International Physical Activity Questionnaire (2011) International Physical Activity Questionnaire (IPAQ). http:// www.ipaq.ki.se/ipaq.htm

39. Haerens L, Deforche B, Maes L, et al. (2007) Physical activity and endurance in normal weight versus overweight boys and girls. J Sport Med Phys Fit 47, 344-350.

40. Krebs-Smith SM, Kott PS \& Guenther PM (1989) Mean proportion and population proportion: two answers to the same question? J Am Diet Assoc 89, 671-676.

41. Sioen I, Matthys C, De Backer G, et al. (2007) Importance of seafood as nutrient source in the diet of Belgian adolescents. J Hum Nutr Diet 20, 580-589.

42. Samuelson G, Bratteby LE, Mohsen R, et al. (2001) Dietary fat intake in healthy adolescents: inverse relationships between the estimated intake of saturated fatty acids and serum cholesterol. Br J Nutr 85, 333-341.

43. Harel Z, Riggs S, Vaz R, et al. (2001) Omega-3 polyunsaturated fatty acids in adolescents: knowledge and consumption. J Adolesc Health 28, 10-15.
44. Ervin RB, Wright JD, Wang CY, et al. (2004) Dietary intake of fats and fatty acids for the United States population: 1999-2000. Advance Data from Vital and Health Statistics, no. 348. Hyattsville, MD: National Center for Health Statistics.

45. Kersting M, Sichert-Hellert W, Alexy U, et al. (1998) Macronutrient intake of 1 to 18 year old German children and adolescents. Z Ernahrungswiss 37, 252-259.

46. Matthys C, De Henauw S, Bellemans M, et al. (2006) Sources of saturated fatty acids in Belgian adolescents' diet: implications for the development of food-based dietary guidelines. Br J Nutr 95, 546-554.

47. Howe P, Meyer B, Record S, et al. (2006) Dietary intake of long-chain omega-3 polyunsaturated fatty acids: contribution of meat sources. Nutrition 22, 47-53.

48. Ramirez-Silva I, Villalpando S, Moreno-Saracho JE, et al. (2011) Fatty acids intake in the Mexican population. Results of the National Nutrition Survey 2006. Nutr Metab (Lond) $\mathbf{8}, 33$.

49. Simopoulos AP (2008) The importance of the omega-6/ omega-3 fatty acid ratio in cardiovascular disease and other chronic diseases. Exp Biol Med (Maywood) 233, 674-688.

50. Deckelbaum RJ \& Calder PC (2010) Dietary $n-3$ and $n-6$ fatty acids: are there 'bad' polyunsaturated fatty acids? Curr Opin Clin Nutr Metab Care 13, 123-124.

51. Stanley JC, Elsom RL, Calder PC, et al. (2007) UK Food Standards Agency Workshop Report: the effects of the dietary $n-6: n-3$ fatty acid ratio on cardiovascular health. Br J Nutr 98, 1305-1310.

52. Holman RT, Johnson SB \& Hatch TF (1982) A case of human linolenic acid deficiency involving neurological abnormalities. Am J Clin Nutr 35, 617-623.

53. Willett WC, Lichtenstein AH, Kuller LH, et al. (2011) The great fat debate: Q \& A. J Am Diet Assoc 111, 672-675.

54. Lichtenstein $\mathrm{AH}$ (2011) The great fat debate: the importance of message translation. J Am Diet Assoc 111, 667-670.

55. Micha R \& Mozaffarian D (2010) Saturated fat and cardiometabolic risk factors, coronary heart disease, stroke, and diabetes: a fresh look at the evidence. Lipids 45, 893-905.

56. Meyer BJ, Mann NJ, Lewis JL, et al. (2003) Dietary intakes and food sources of omega- 6 and omega-3 polyunsaturated fatty acids. Lipids 38, 391-398.

57. Ollis TE, Meyer BJ \& Howe PR (1999) Australian food sources and intakes of omega- 6 and omega-3 polyunsaturated fatty acids. Ann Nutr Metab 43, 346-355.

58. Monge-Rojas R, Campos H \& Fernández Rojas X (2005) Saturated and cis- and trans-unsaturated fatty acids intake in rural and urban Costa Rican adolescents. J Am Coll Nutr 24, 286-293.

59. Max Rubner-Institut (2011) Bundeslebensmittelschlüssel (BLS): German Nutrient Database. http://bls.nvs2.de

60. Deharveng G, Charrondiere UR, Slimani N, et al. (1999) Comparison of nutrients in the food composition tables available in the nine European countries participating in EPIC. European Prospective Investigation into Cancer and Nutrition. Eur J Clin Nutr 53, 60-79. 